\title{
AN ASSEMBLAGE-THEORETIC PROOF OF THE EXISTENCE OF TRANSCENDENTALLY TRANSCENDENTAL FUNCTIONS*
}

\author{
BY J. F. RITT AND ELI GOURIN
}

1. Introduction. The first example of a transcendentally transcendental function, $\dagger$ that is, of an analytic function not a solution of any algebraic differential equation, was given by Hölder, $\ddagger$ who, in 1887 , showed that the gamma function does not satisfy any such equation. Other investigations have followed Hölder's. In some of these, functional equations of different types are studied, to find which of their solutions satisfy algebraic differential equations. In others, e.g., in that of Hurwitz, on power series with rational coefficients $\S$ and in that of Ostrowsky on Dirichlet series, $\|$ certain analytic expressions are examined and conditions found under which they represent transcendentally transcendental functions.

In the present note, the existence of transcendentally transcendental functions is shown on an a priori basis, by a proof resembling that proof of the existence of transcendental numbers which is based on the countability of the algebraic numbers. The totality of algebraic differential equations has the same power as the totality of analytic functions, but there exists a countable set of the equations, namely, those with integral coefficients, whose solutions form the totality of the solutions of all algebraic differential equations. It follows that the solutions of the countable set of equations do not exhaust the analytic functions.

* Presented to the Society, December 29, 1926.

$\dagger$ This term is due to E. H. Moore, Mathematische Annalen, vol. 48 (1896), p. 49.

$\ddagger$ Mathematische Annalen, vol. 28 (1887), p. 1.

$\S$ Annales de l'Ecole Normale Supérieure, vol. 6 (1889), p. 327.

|| Mathematische Zeitschrift, vol. 8 (1920), p. 241. 
Our result throws some light on the distribution of transcendentally transcendental functions among the analytic functions. We prove that it is possible to choose the coefficients in the series

$$
a_{0}+a_{1} x+\cdots+a_{n} x^{n}+\cdots
$$

so that the series does not satisfy formally any algebraic differential equation, with the following degree of freedom:

$a_{0}$ may be given any value not on a certain countable set;

$a_{1}$ may then be given any value not on a certain countable set which depends on the choice of $a_{0}$;

$a_{n}$ may be given any value not on a certain countable set which depends on the choices of $a_{0}, \cdots, a_{n-1}$;

Any set of $a$ 's which do not increase too rapidly give a transcendentally transcendental function.

2. Algebraic Differential Equations. Let $y(x)$ be any function satisfying an algebraic differential equation. Representing by $y_{p}$ the $p$ th derivative of $y$, we write the equation for $y$ in the form

$$
\sum c_{(i)} x^{i} y^{i_{0}} y_{1} i_{1} \cdots y_{n}^{i_{n}}=0 .
$$

Here each $c$ is a constant not zero. It is understood that the expressions $x^{i} \cdots y_{n}{ }^{i_{n}}$ are distinct from one another.

Now (1) states that, for the given function $y(x)$, the expressions $x^{i} \cdots y_{n}{ }^{i}{ }_{n}$ are linearly dependent. Thus, if we set the wronskian of these expressions equal to zero, we shall have an algebraic differential equation for $y(x)$, usually of order greater than $n$, with integral coefficients. All that it is necessary to show is that the wronskian does not vanish identically in $x \cdots y_{n}$. If it did, every function with $n$ derivatives would satisfy an equation like (1), that is, an equation with the same expressions $x^{i} \cdots y_{n}{ }^{i_{n}}$ as appear in (1), with constants $c$ not all zero. But because we can construct a function with any given values for itself and for its first $n$ derivatives at any number of points, it 
would follow easily that an equation like (1) exists, with coefficients $c$ not all zero, which is an identity for $x, \cdots, y_{n}$ arbitrary. This is absurd. We know thus that $y(x)$ satisfies an algebraic differential equation with integral coefficients.*

3. Transcendentally Transcendental Functions. The algebraic differential equations with integral coefficients are countable. Let them be arranged in a sequence

$$
e_{1}=0, \quad e_{2}=0, \cdots, \quad e_{n}=0, \cdots .
$$

If the polynomial $e_{i}$ vanishes when $x=h$, for all values of the variables other than $x$ which appear in $e_{i}$, then $e_{i}$ is divisible by $x-h$. As each $e_{i}$ has only a finite number of factors of the form $x-h$, the polynomials of (2) have, collectively, only a countable set of such factors.

If we replace $x$ in (2) by any value distinct from the $h$ 's of the countable set just mentioned, the equations of (2) become non-identical equations which do not involve $x$,

$$
e_{1}^{\prime}=0, \quad e_{2}^{\prime}=0, \cdots, \quad e_{n}^{\prime}=0, \cdots .
$$

Of course, an $e_{i}^{\prime}$ may be a constant. This simply means that $e_{i}=0$ has no solution analytic for the chosen value of $h$.

We can now choose $y$ anywhere, except on a countable set, in such a way as to reduce the equations of (3) to nonidentical equations involving neither $x$ nor $y$, and can continue in the same fashion for $y_{1}$, etc. We find thus a set of values of our variables which satisfy no equation (2).

If the values chosen for $x, y, y_{1}$, etc., are respectively $h$, $k_{0}, k_{1}$, etc., the power series $\sum k_{n}(x-h)^{n} / n$ ! will not satisfy formally any algebraic differential equation. The series $\sum k_{n} x^{n} / n$ ! cannot satisfy such an equation either. This proves the result stated in the introduction.

Col umbia University

* Hurwitz, loc. cit., states without proof that a power series with rational coefficients which satisfies an algebraic differential equation, satisfies such an equation with integral coefficients. Very probably he had the more general result just proved in mind. In any case, the countability of the equations with integral coefficients plays no role in Hurwitz's work. 